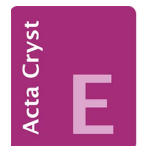

OPEN $\curvearrowright$ ACCESS COMMUNICATIONS

ISSN 2056-9890

\section{Crystal structure of 1,3,5-trimethyl-2,4- dinitrobenzene}

\section{Ouarda Brihi, ${ }^{a} *$ Noudjoud Hamdouni, ${ }^{a}$ Ali Boudjada ${ }^{a}$ and Jean Meinnel ${ }^{b}$}

aLaboratoire de Cristallographie, Département de Physique, Université MentouriConstantine, 25000 Constantine, Algeria, and ${ }^{\mathbf{b}}$ UMR 6226 CNRS-Université Rennes 1 'Sciences Chimiques de Rennes', Equipe 'Matière Condensée et Systèmes Electroactifs', 263 Avenue du Général Leclerc, F-35042 Rennes, France. *Correspondence e-mail: ouardabrihi@yahoo.fr

Received 7 July 2015; accepted 28 July 2015

Edited by W. T. A. Harrison, University of Aberdeen, Scotland

In the title compound, $\mathrm{C}_{9} \mathrm{H}_{10} \mathrm{~N}_{2} \mathrm{O}_{4}$, the planes of the nitro groups subtend dihedral angles of $55.04(15)$ and $63.23(15)^{\circ}$ with that of the aromatic ring. These tilts are in opposite senses and the molecule possesses approximate mirror symmetry about a plane normal to the molecule. In the crystal, molecules form stacks in the [100] direction with adjacent molecules related by translation, although the centroid-centroid separation of 4.136 (5) $\AA$ is probably too long to regard as a significant aromatic $\pi-\pi$ stacking interaction. An extremely weak $\mathrm{C}-\mathrm{H} \cdots \mathrm{O}$ interaction is also present.

Keywords: crystal structure; dinitrobenzene; weak $\mathrm{C}-\mathrm{H} \ldots \mathrm{O}$ interaction.

CCDC reference: 1415489

\section{Related literature}

For the structures and properties of related compounds, see:

Tazi et al. (1995); Hernandez et al. (2003).

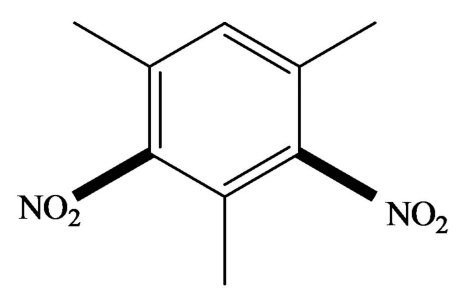

\section{Experimental}

2.1. Crystal data

$\mathrm{C}_{9} \mathrm{H}_{10} \mathrm{~N}_{2} \mathrm{O}_{4}$

$M_{r}=210.19$

Orthorhombic, $P 2_{1} 2_{1} 2_{1}$

$a=4.136(5) \AA$

$b=13.916(5) \AA$

$c=17.194(5) \AA$

$$
\begin{aligned}
& V=989.6(13) \AA^{3} \\
& Z=4 \\
& \text { Mo } K \alpha \text { radiation } \\
& \mu=0.11 \mathrm{~mm}^{-1} \\
& T=293 \mathrm{~K} \\
& 0.1 \times 0.08 \times 0.08 \mathrm{~mm}
\end{aligned}
$$

\subsection{Data collection \\ Oxford Diffraction Xcalibur diffractometer tion, 2010)

$$
T_{\text {min }}=0.618, T_{\text {max }}=1.000
$$ \\ 2.3. Refinement \\ $R\left[F^{2}>2 \sigma\left(F^{2}\right)\right]=0.053$ \\ $w R\left(F^{2}\right)=0.113$ \\ $S=0.93$ \\ 2730 reflections \\ 139 parameters}

Absorption correction: multi-scan (CrysAlis PRO; Oxford Diffrac-

3941 measured reflections 2730 independent reflections 1302 reflections with $I>2 \sigma(I)$ $R_{\text {int }}=0.032$ Standard reflections: ?
$\mathrm{H}$ atoms treated by a mixture of independent and constrained refinement
$\Delta \rho_{\max }=0.12{\mathrm{e} \AA^{-3}}^{-3}$
$\Delta \rho_{\min }=-0.15$ e $\AA^{-3}$

Table 1

Hydrogen-bond geometry $\left(\AA{ }^{\circ}\right)$.

\begin{tabular}{lllll}
\hline$D-\mathrm{H} \cdots A$ & $D-\mathrm{H}$ & $\mathrm{H} \cdots A$ & $D \cdots A$ & $D-\mathrm{H} \cdots A$ \\
\hline $\mathrm{C} 7-\mathrm{H} 7 C \cdots \mathrm{O}^{\mathrm{i}}$ & 0.96 & 2.60 & $3.232(4)$ & 124 \\
\hline
\end{tabular}

Symmetry code: (i) $x-\frac{1}{2},-y+\frac{1}{2},-z+1$.

Data collection: CrysAlis RED (Oxford Diffraction, 2002); cell refinement: CrysAlis RED; data reduction: CrysAlis RED; program(s) used to solve structure: SIR2002 (Burla et al., 2005); program(s) used to refine structure: SHELXL97 (Sheldrick, 2008); molecular graphics: CAMERON (Watkin et al., 1996); software used to prepare material for publication: WinGX (Farrugia, 2012).

Supporting information for this paper is available from the IUCr electronic archives (Reference: HB7463).

\section{References}

Burla, M. C., Caliandro, R., Camalli, M., Carrozzini, B., Cascarano, G. L., De Caro, L., Giacovazzo, C., Polidori, G. \& Spagna, R. (2005). J. Appl. Cryst. 38, 381-388.

Farrugia, L. J. (2012). J. Appl. Cryst. 45, 849-854.

Hernandez, O., Cousson, A., Plazanet, M., Nierlich, M. \& Meinnel, J. (2003). Acta Cryst. C59, o445-0450. 


\section{data reports}

Oxford Diffraction (2002). CrysAlis RED. Oxford Diffraction Ltd, Abingdon, England.

Oxford Diffraction (2010). CrysAlis PRO. Oxford Diffraction Ltd, Abingdon, England.

Sheldrick, G. M. (2008). Acta Cryst. A64, 112-122.
Tazi, M., Meinnel, J., Sanquer, M., Nusimovici, M., Tonnard, F. \& Carrie, R. (1995). Acta Cryst. B51, 838-847.

Watkin, D. J., Prout, C. K. \& Pearce, L. J. (1996). CAMERON. Chemical Crystallography Laboratory, Oxford, England. 


\section{supporting information}

Acta Cryst. (2015). E71, o670-o671 [https://doi.org/10.1107/S2056989015014243]

\section{Crystal structure of 1,3,5-trimethyl-2,4-dinitrobenzene}

\section{Ouarda Brihi, Noudjoud Hamdouni, Ali Boudjada and Jean Meinnel}

\section{S1. Experimental}

The commercially available compound (Sigma-Aldrich) Was recrystallized from ethanol solution.

\section{S2. Refinement}

All non-H atoms were refined with anisotropic atomic displacement parameters. All $\mathrm{H}$ atoms were localized in a Fourier maps but introduced in calculated positions and treated as riding on their parent $\mathrm{C}$ atoms with $\mathrm{C}_{\text {aryl }}-\mathrm{H}_{\text {ary }}=0.93 \AA ; \mathrm{C}_{\text {methyl }}$ $-\mathrm{H}_{\text {methyl }}=0.96 \AA$ and $U_{\text {iso }}\left(\mathrm{H}_{\text {methyl }}\right)=1.5 U_{\text {eq }}\left(\mathrm{C}_{\text {methyl }}\right)$ or $U_{\text {iso }}\left(\mathrm{H}_{\text {aryl }}\right)=1.2 U_{\text {eq }}\left(\mathrm{C}_{\text {aryl }}\right)$. The atoms of benzene cycle present parameters of atomic displacements weaker than those of the substituent atoms.

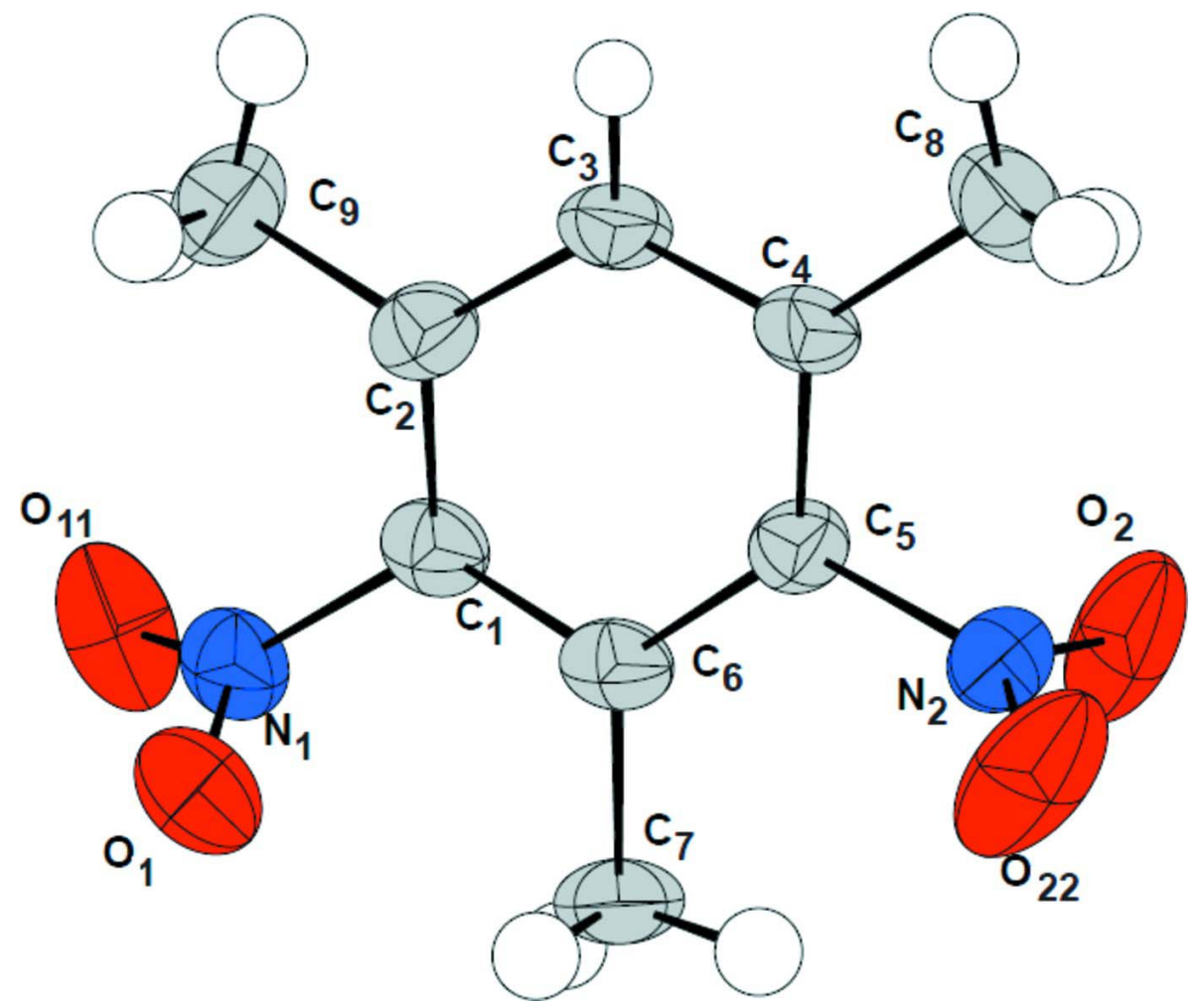

Figure 1

The molecular structure of (I) with displacement ellipsoids drawn at the 50\% probability level. 


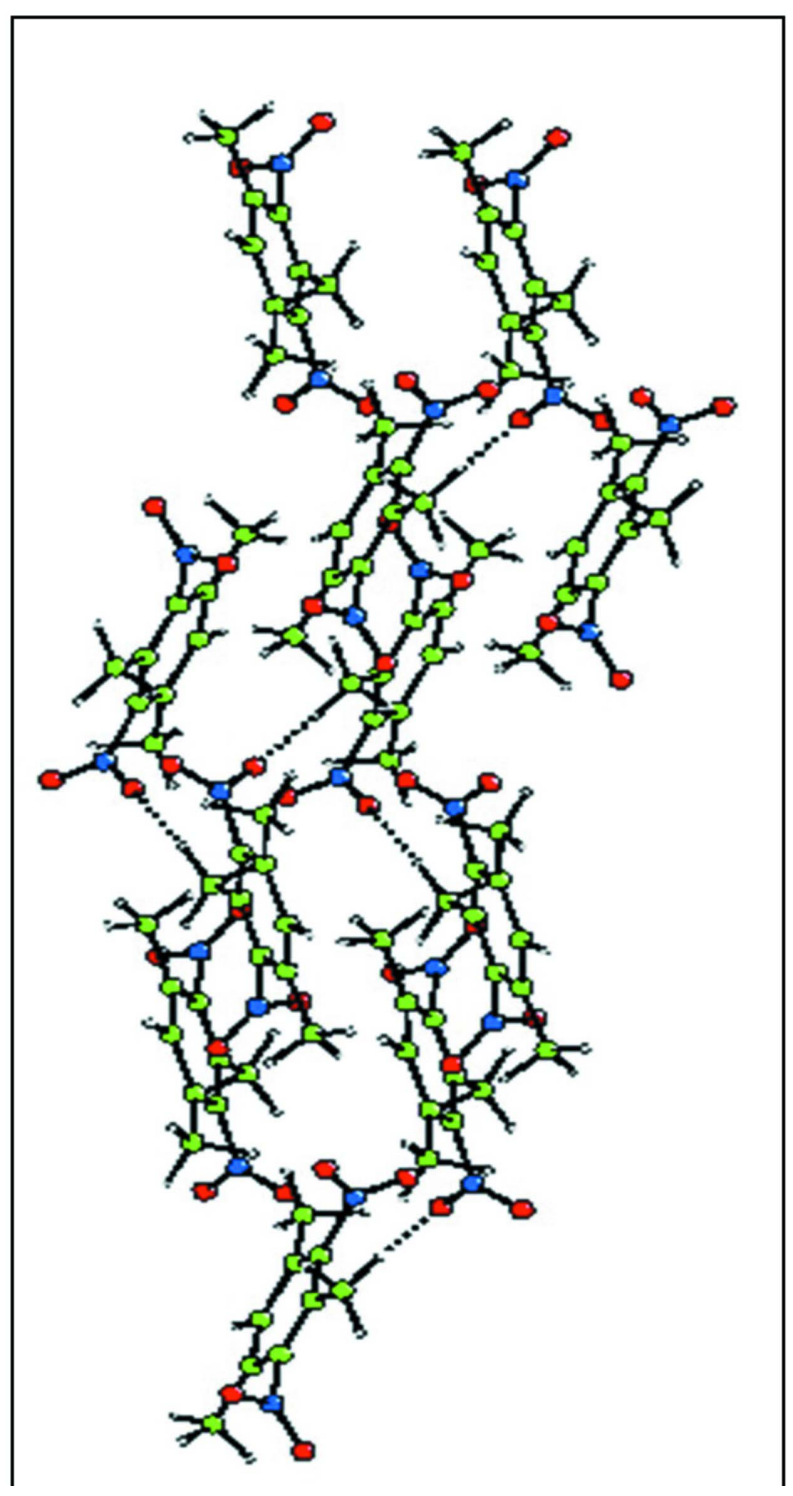

Figure 2

The crystal packing of (I) at $293 \mathrm{~K}$, along the $b$ axis. 


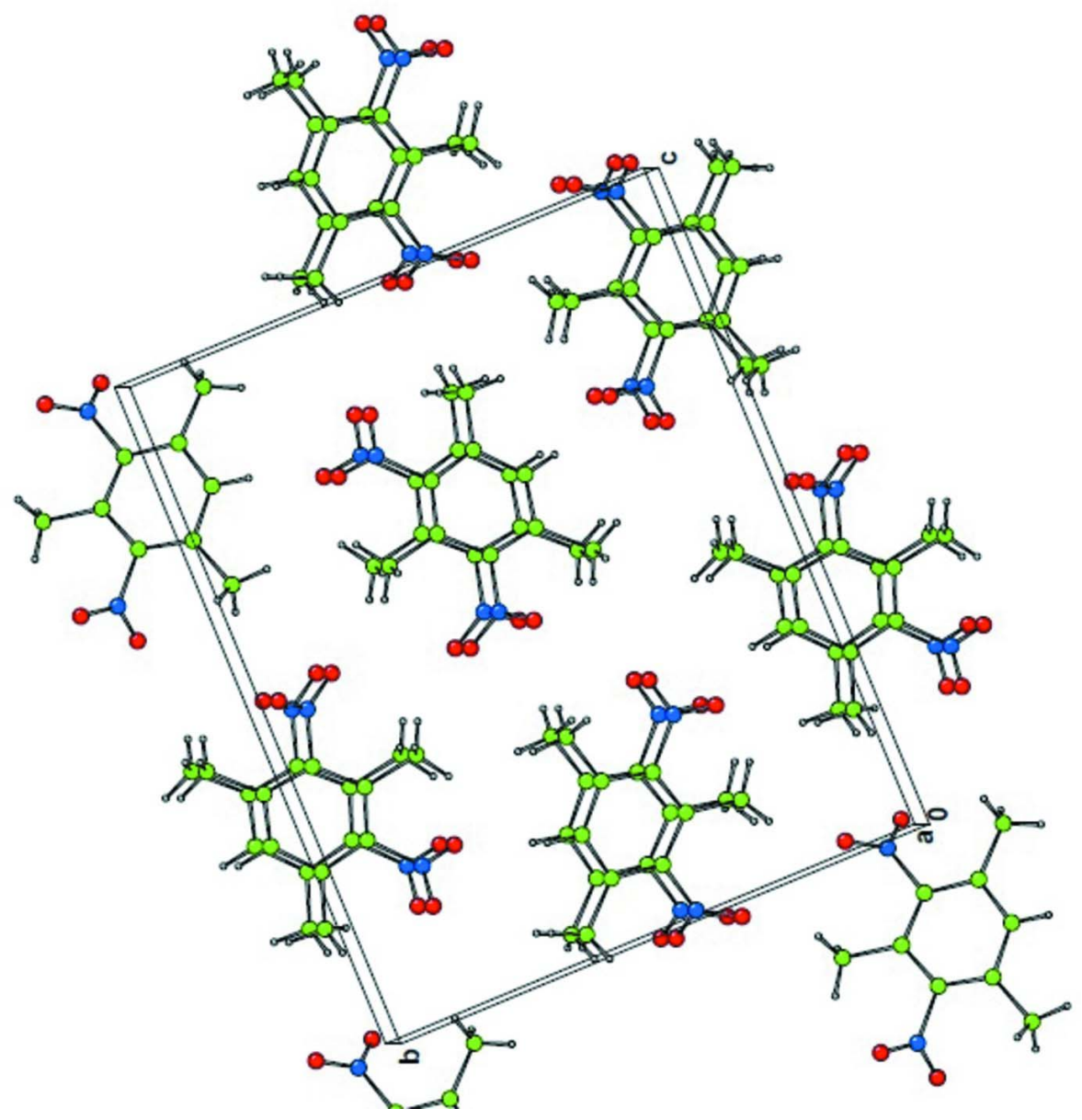

Figure 3

The crystal packing of (I) at $293 \mathrm{~K}$, according to the direction [100].

\section{1,3,5-Trimethyl-2,4-dinitrobenzene}

\section{Crystal data}

$\mathrm{C}_{9} \mathrm{H}_{10} \mathrm{~N}_{2} \mathrm{O}_{4}$

$M_{r}=210.19$

Orthorhombic, $P 2_{1} 2_{1} 2_{1}$

Hall symbol: P 2ac 2ab

$a=4.136(5) \AA$

$b=13.916(5) \AA$

$c=17.194(5) \AA$

$V=989.6(13) \AA^{3}$

$Z=4$

\section{Data collection}

Oxford Diffraction Xcalibur diffractometer

Radiation source: Enhance (Mo) X-ray Source Graphite monochromator CCD scans
$F(000)=440$

$D_{\mathrm{x}}=1.411 \mathrm{Mg} \mathrm{m}^{-3}$

Mo $K \alpha$ radiation, $\lambda=0.71073 \AA$

Cell parameters from 1062 reflections

$\theta=3.8-25.0^{\circ}$

$\mu=0.11 \mathrm{~mm}^{-1}$

$T=293 \mathrm{~K}$

Needle, colourless

$0.1 \times 0.08 \times 0.08 \mathrm{~mm}$

Absorption correction: multi-scan

(CrysAlis PRO; Oxford Diffraction, 2010)

$T_{\min }=0.618, T_{\max }=1.000$

3941 measured reflections

2730 independent reflections

1302 reflections with $I>2 \sigma(I)$ 


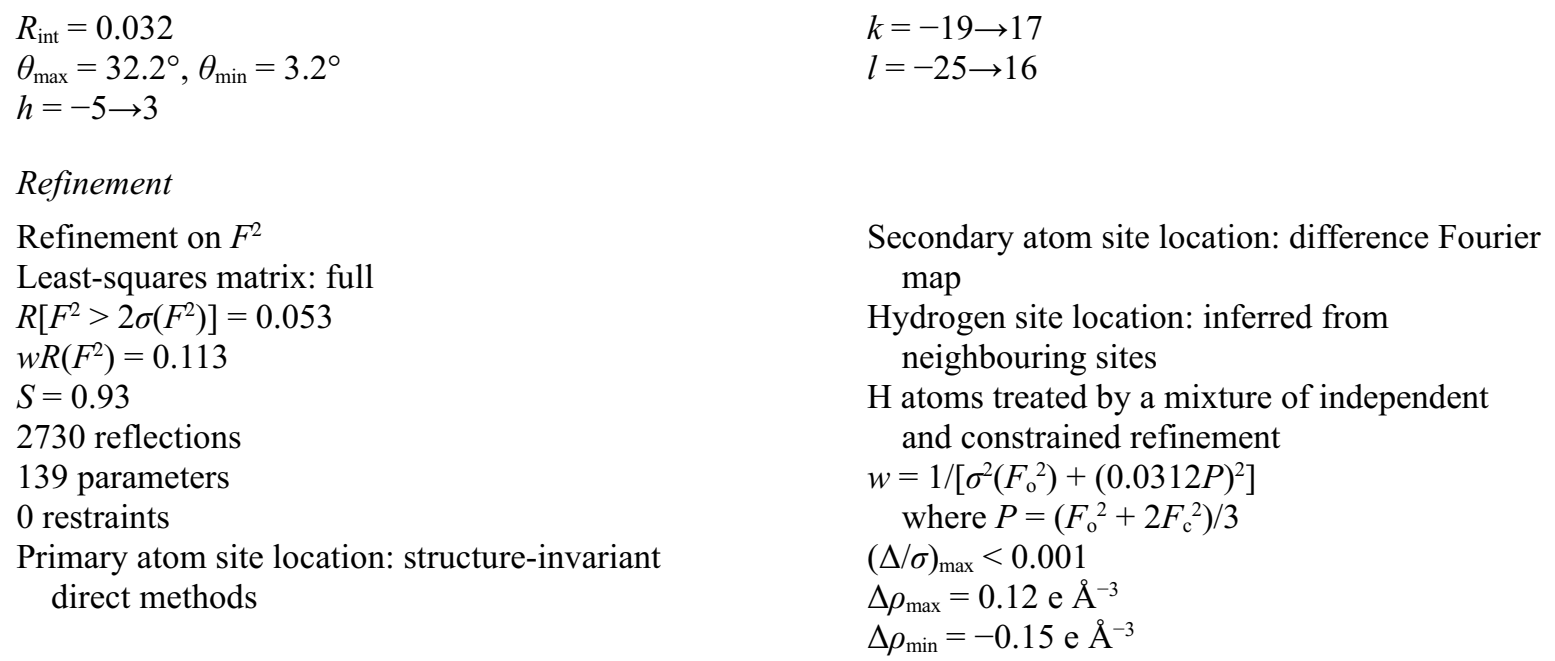

\section{Special details}

Experimental. Absorption correction: CrysAlisPro, Empirical absorption correction using spherical harmonics, implemented in SCALE3 ABSPACK scaling algorithm.

Geometry. Bond distances, angles etc. have been calculated using the rounded fractional coordinates. All su's are estimated from the variances of the (full) variance-covariance matrix. The cell e.s.d.'s are taken into account in the estimation of distances, angles and torsion angles

Refinement. Refinement of $F^{2}$ against ALL reflections. The weighted $R$-factor $w R$ and goodness of fit $S$ are based on $F^{2}$, conventional $R$-factors $R$ are based on $F$, with $F$ set to zero for negative $F^{2}$. The threshold expression of $F^{2}>\sigma\left(F^{2}\right)$ is used only for calculating $R$-factors (gt) etc. and is not relevant to the choice of reflections for refinement. $R$-factors based on $F^{2}$ are statistically about twice as large as those based on $F$, and $R$-factors based on ALL data will be even larger.

Fractional atomic coordinates and isotropic or equivalent isotropic displacement parameters $\left(\AA^{2}\right)$

\begin{tabular}{lllll}
\hline & $x$ & $y$ & $z$ & $U_{\text {iso }} * / U_{\text {eq }}$ \\
\hline O1 & $0.5095(6)$ & $0.13682(16)$ & $0.51948(12)$ & $0.0830(9)$ \\
O2 & $0.4780(6)$ & $0.14379(17)$ & $0.16111(14)$ & $0.0975(10)$ \\
O11 & $0.1589(6)$ & $0.0227(2)$ & $0.51482(12)$ & $0.0930(9)$ \\
O22 & $0.8082(7)$ & $0.22717(18)$ & $0.22671(15)$ & $0.1057(11)$ \\
$\mathrm{N} 1$ & $0.3785(6)$ & $0.06911(19)$ & $0.48712(13)$ & $0.0612(9)$ \\
$\mathrm{N} 2$ & $0.6374(6)$ & $0.15688(17)$ & $0.21810(14)$ & $0.0576(8)$ \\
C1 & $0.4961(5)$ & $0.04214(19)$ & $0.40898(13)$ & $0.0446(8)$ \\
C2 & $0.5978(6)$ & $-0.05263(18)$ & $0.39734(15)$ & $0.0482(8)$ \\
C3 & $0.7215(6)$ & $-0.07338(17)$ & $0.32487(16)$ & $0.0501(9)$ \\
C4 & $0.7380(6)$ & $-0.00732(19)$ & $0.26478(14)$ & $0.0466(8)$ \\
C5 & $0.6263(6)$ & $0.08533(17)$ & $0.28156(15)$ & $0.0439(8)$ \\
C6 & $0.5084(5)$ & $0.11344(17)$ & $0.35299(14)$ & $0.0435(8)$ \\
C7 & $0.3848(8)$ & $0.21448(18)$ & $0.36780(16)$ & $0.0664(11)$ \\
C8 & $0.8759(7)$ & $-0.0350(2)$ & $0.18697(15)$ & $0.0632(10)$ \\
C9 & $0.5834(7)$ & $-0.1295(2)$ & $0.45897(17)$ & $0.0701(11)$ \\
H3 & 0.79817 & -0.13520 & 0.31580 & $0.0601 *$ \\
H7A & 0.30028 & 0.24079 & 0.32036 & $0.0995^{*}$ \\
H7B & 0.55903 & 0.25404 & 0.38611 & $0.0995 *$ \\
H7C & 0.21682 & 0.21253 & 0.40631 & $0.0995 *$ \\
H8A & 1.00516 & 0.01682 & 0.16721 & $0.0947 *$
\end{tabular}




\begin{tabular}{lllll} 
H8B & 0.70269 & -0.04818 & 0.15135 & $0.0947^{*}$ \\
H8C & 1.00773 & -0.09134 & 0.19269 & $0.0947^{*}$ \\
H9A & 0.66925 & -0.10482 & 0.50682 & $0.1050^{*}$ \\
H9B & 0.70885 & -0.18397 & 0.44265 & $0.1050^{*}$ \\
H9C & 0.36286 & -0.14884 & 0.46668 & $0.1050^{*}$ \\
\hline
\end{tabular}

Atomic displacement parameters $\left(\AA^{2}\right)$

\begin{tabular}{lllllll}
\hline & $U^{11}$ & $U^{22}$ & $U^{33}$ & $U^{12}$ & $U^{13}$ & $U^{23}$ \\
\hline O1 & $0.1139(17)$ & $0.0722(16)$ & $0.0629(13)$ & $-0.0037(15)$ & $-0.0125(13)$ & $-0.0276(12)$ \\
O2 & $0.130(2)$ & $0.0873(18)$ & $0.0751(14)$ & $-0.0364(15)$ & $-0.0386(16)$ & $0.0295(14)$ \\
O11 & $0.0756(14)$ & $0.129(2)$ & $0.0743(14)$ & $-0.0118(16)$ & $0.0166(12)$ & $-0.0084(15)$ \\
O22 & $0.126(2)$ & $0.0670(16)$ & $0.124(2)$ & $-0.0504(15)$ & $-0.0339(17)$ & $0.0323(15)$ \\
N1 & $0.0600(14)$ & $0.0690(18)$ & $0.0547(14)$ & $0.0088(14)$ & $-0.0066(13)$ & $-0.0055(14)$ \\
N2 & $0.0685(15)$ & $0.0394(13)$ & $0.0648(15)$ & $-0.0027(12)$ & $-0.0067(14)$ & $0.0032(13)$ \\
C1 & $0.0449(13)$ & $0.0466(14)$ & $0.0424(13)$ & $-0.0009(12)$ & $-0.0070(12)$ & $-0.0089(12)$ \\
C2 & $0.0508(14)$ & $0.0394(14)$ & $0.0545(15)$ & $-0.0036(12)$ & $-0.0098(13)$ & $-0.0029(13)$ \\
C3 & $0.0570(16)$ & $0.0294(12)$ & $0.0639(17)$ & $0.0000(11)$ & $-0.0025(14)$ & $-0.0069(13)$ \\
C4 & $0.0507(14)$ & $0.0362(14)$ & $0.0529(14)$ & $-0.0032(11)$ & $-0.0020(12)$ & $-0.0082(13)$ \\
C5 & $0.0477(13)$ & $0.0327(12)$ & $0.0513(15)$ & $-0.0047(11)$ & $-0.0106(13)$ & $0.0033(12)$ \\
C6 & $0.0437(13)$ & $0.0353(12)$ & $0.0515(15)$ & $0.0042(11)$ & $-0.0110(12)$ & $-0.0090(12)$ \\
C7 & $0.083(2)$ & $0.0434(16)$ & $0.0727(19)$ & $0.0206(15)$ & $-0.0153(16)$ & $-0.0123(15)$ \\
C8 & $0.0709(19)$ & $0.0503(16)$ & $0.0683(17)$ & $-0.0045(15)$ & $0.0121(15)$ & $-0.0125(15)$ \\
C9 & $0.082(2)$ & $0.0544(19)$ & $0.074(2)$ & $-0.0040(18)$ & $-0.0028(16)$ & $0.0144(16)$ \\
& & & & & & \\
\hline
\end{tabular}

Geometric parameters $\left(\AA,{ }^{\circ}\right)$

\begin{tabular}{llll}
\hline $\mathrm{O} 1-\mathrm{N} 1$ & $1.221(3)$ & $\mathrm{C} 5-\mathrm{C} 6$ & $1.378(3)$ \\
$\mathrm{O} 2-\mathrm{N} 2$ & $1.195(3)$ & $\mathrm{C} 6-\mathrm{C} 7$ & $1.518(4)$ \\
$\mathrm{O} 11-\mathrm{N} 1$ & $1.212(4)$ & $\mathrm{C} 3-\mathrm{H} 3$ & 0.9300 \\
$\mathrm{O} 22-\mathrm{N} 2$ & $1.216(4)$ & $\mathrm{C} 7-\mathrm{H} 7 \mathrm{~A}$ & 0.9600 \\
$\mathrm{~N} 1-\mathrm{C} 1$ & $1.477(3)$ & $\mathrm{C} 7-\mathrm{H} 7 \mathrm{~B}$ & 0.9600 \\
$\mathrm{~N} 2-\mathrm{C} 5$ & $1.478(3)$ & $\mathrm{C} 7-\mathrm{H} 7 \mathrm{C}$ & 0.9600 \\
$\mathrm{C} 1-\mathrm{C} 2$ & $1.399(4)$ & $\mathrm{C} 8-\mathrm{H} 8 \mathrm{~A}$ & 0.9600 \\
$\mathrm{C} 1-\mathrm{C} 6$ & $1.383(3)$ & $\mathrm{C} 8-\mathrm{H} 8 \mathrm{~B}$ & 0.9600 \\
$\mathrm{C} 2-\mathrm{C} 3$ & $1.378(4)$ & $\mathrm{C} 8-\mathrm{H} 8 \mathrm{C}$ & 0.9600 \\
$\mathrm{C} 2-\mathrm{C} 9$ & $1.507(4)$ & $\mathrm{C} 9-\mathrm{H} 9 \mathrm{~A}$ & 0.9600 \\
$\mathrm{C} 3-\mathrm{C} 4$ & $1.385(4)$ & $\mathrm{C} 9-\mathrm{H} 9 \mathrm{~B}$ & 0.9600 \\
$\mathrm{C} 4-\mathrm{C} 5$ & $1.400(4)$ & $\mathrm{C} 9-\mathrm{H} 9 \mathrm{C}$ & \\
$\mathrm{C} 4-\mathrm{C} 8$ & $1.505(4)$ & & $122.1(2)$ \\
& & & 118.00 \\
$\mathrm{O} 1-\mathrm{N} 1-\mathrm{O} 11$ & $124.4(2)$ & $\mathrm{C} 5-\mathrm{C} 6-\mathrm{C} 7$ & 118.00 \\
$\mathrm{O} 1-\mathrm{N} 1-\mathrm{C} 1$ & $117.7(2)$ & $\mathrm{C} 2-\mathrm{C} 3-\mathrm{H} 3$ & 109.00 \\
$\mathrm{O} 11-\mathrm{N} 1-\mathrm{C} 1$ & $118.0(2)$ & $\mathrm{C} 4-\mathrm{C} 3-\mathrm{H} 3$ & 109.00 \\
$\mathrm{O} 2-\mathrm{N} 2-\mathrm{O} 22$ & $122.9(3)$ & $\mathrm{C} 6-\mathrm{C} 7-\mathrm{H} 7 \mathrm{~A}$ & 109.00 \\
$\mathrm{O} 2-\mathrm{N} 2-\mathrm{C} 5$ & $119.1(2)$ & $\mathrm{C} 6-\mathrm{C} 7-\mathrm{H} 7 \mathrm{~B}$ & 109.00 \\
$\mathrm{O} 22-\mathrm{N} 2-\mathrm{C} 5$ & $118.1(2)$ & $\mathrm{C} 6-\mathrm{C} 7-\mathrm{H} 7 \mathrm{C}$ & \\
$\mathrm{N} 1-\mathrm{C} 1-\mathrm{C} 2$ & $118.0(2)$ & $\mathrm{H} 7 \mathrm{~A}-\mathrm{C} 7-\mathrm{H} 7 \mathrm{~B}$ &
\end{tabular}




$\begin{array}{ll}\mathrm{N} 1-\mathrm{C} 1-\mathrm{C} 6 & 117.6(2) \\ \mathrm{C} 2-\mathrm{C} 1-\mathrm{C} 6 & 124.5(2) \\ \mathrm{C} 1-\mathrm{C} 2-\mathrm{C} 3 & 116.0(2) \\ \mathrm{C} 1-\mathrm{C} 2-\mathrm{C} 9 & 123.8(2) \\ \mathrm{C} 3-\mathrm{C} 2-\mathrm{C} 9 & 120.1(2) \\ \mathrm{C} 2-\mathrm{C} 3-\mathrm{C} 4 & 123.7(2) \\ \mathrm{C} 3-\mathrm{C} 4-\mathrm{C} 5 & 116.2(2) \\ \mathrm{C} 3-\mathrm{C} 4-\mathrm{C} 8 & 120.8(2) \\ \mathrm{C} 5-\mathrm{C} 4-\mathrm{C} 8 & 123.0(2) \\ \mathrm{N} 2-\mathrm{C} 5-\mathrm{C} 4 & 117.3(2) \\ \mathrm{N} 2-\mathrm{C} 5-\mathrm{C} 6 & 118.5(2) \\ \mathrm{C} 4-\mathrm{C} 5-\mathrm{C} 6 & 124.2(2) \\ \mathrm{C} 1-\mathrm{C} 6-\mathrm{C} 5 & 115.4(2) \\ \mathrm{C} 1-\mathrm{C} 6-\mathrm{C} 7 & 122.4(2) \\ & \\ \mathrm{O} 1-\mathrm{N} 1-\mathrm{C} 1-\mathrm{C} 2 & 124.4(3) \\ \mathrm{O} 11-\mathrm{N} 1-\mathrm{C} 1-\mathrm{C} 2 & -55.6(3) \\ \mathrm{O} 1-\mathrm{N} 1-\mathrm{C} 1-\mathrm{C} 6 & -53.5(3) \\ \mathrm{O} 11-\mathrm{N} 1-\mathrm{C} 1-\mathrm{C} 6 & 126.6(3) \\ \mathrm{O} 2-\mathrm{N} 2-\mathrm{C} 5-\mathrm{C} 6 & -116.9(3) \\ \mathrm{O} 2-\mathrm{N} 2-\mathrm{C} 5-\mathrm{C} 4 & 63.2(3) \\ \mathrm{O} 22-\mathrm{N} 2-\mathrm{C} 5-\mathrm{C} 4 & -117.2(3) \\ \mathrm{O} 22-\mathrm{N} 2-\mathrm{C} 5-\mathrm{C} 6 & 62.7(3) \\ \mathrm{N} 1-\mathrm{C} 1-\mathrm{C} 2-\mathrm{C} 3 & -176.7(2) \\ \mathrm{N} 1-\mathrm{C} 1-\mathrm{C} 6-\mathrm{C} 7 & -4.4(3) \\ \mathrm{C} 2-\mathrm{C} 1-\mathrm{C} 6-\mathrm{C} 5 & 1.0(3) \\ \mathrm{C} 2-\mathrm{C} 1-\mathrm{C} 6-\mathrm{C} 7 & 178.0(2) \\ \mathrm{C} 6-\mathrm{C} 1-\mathrm{C} 2-\mathrm{C} 9 & 179.9(2) \\ \mathrm{N} 1-\mathrm{C} 1-\mathrm{C} 2-\mathrm{C} 9 & 2.2(4) \\ & \end{array}$

$\mathrm{H} 7 \mathrm{~A}-\mathrm{C} 7-\mathrm{H} 7 \mathrm{C}$

H7B-C7-H7C

$\mathrm{C} 4-\mathrm{C} 8-\mathrm{H} 8 \mathrm{~A}$

$\mathrm{C} 4-\mathrm{C} 8-\mathrm{H} 8 \mathrm{~B}$

$\mathrm{C} 4-\mathrm{C} 8-\mathrm{H} 8 \mathrm{C}$

$\mathrm{H} 8 \mathrm{~A}-\mathrm{C} 8-\mathrm{H} 8 \mathrm{~B}$

$\mathrm{H} 8 \mathrm{~A}-\mathrm{C} 8-\mathrm{H} 8 \mathrm{C}$

$\mathrm{H} 8 \mathrm{~B}-\mathrm{C} 8-\mathrm{H} 8 \mathrm{C}$

$\mathrm{C} 2-\mathrm{C} 9-\mathrm{H} 9 \mathrm{~A}$

$\mathrm{C} 2-\mathrm{C} 9-\mathrm{H} 9 \mathrm{~B}$

$\mathrm{C} 2-\mathrm{C} 9-\mathrm{H} 9 \mathrm{C}$

H9A-C9- H9B

H9A-C9-H9C

H9B $-\mathrm{C} 9-\mathrm{H} 9 \mathrm{C}$

$\mathrm{C} 6-\mathrm{C} 1-\mathrm{C} 2-\mathrm{C} 3$

$\mathrm{N} 1-\mathrm{C} 1-\mathrm{C} 6-\mathrm{C} 5$

$\mathrm{C} 1-\mathrm{C} 2-\mathrm{C} 3-\mathrm{C} 4$

$\mathrm{C} 9-\mathrm{C} 2-\mathrm{C} 3-\mathrm{C} 4$

$\mathrm{C} 2-\mathrm{C} 3-\mathrm{C} 4-\mathrm{C} 5$

$\mathrm{C} 2-\mathrm{C} 3-\mathrm{C} 4-\mathrm{C} 8$

$\mathrm{C} 3-\mathrm{C} 4-\mathrm{C} 5-\mathrm{C} 6$

$\mathrm{C} 8-\mathrm{C} 4-\mathrm{C} 5-\mathrm{N} 2$

$\mathrm{C} 8-\mathrm{C} 4-\mathrm{C} 5-\mathrm{C} 6$

$\mathrm{C} 3-\mathrm{C} 4-\mathrm{C} 5-\mathrm{N} 2$

$\mathrm{N} 2-\mathrm{C} 5-\mathrm{C} 6-\mathrm{C} 1$

$\mathrm{N} 2-\mathrm{C} 5-\mathrm{C} 6-\mathrm{C} 7$

$\mathrm{C} 4-\mathrm{C} 5-\mathrm{C} 6-\mathrm{C} 1$

$\mathrm{C} 4-\mathrm{C} 5-\mathrm{C} 6-\mathrm{C} 7$
109.00
109.00
109.00
109.00
109.00
109.00
109.00
109.00
110.00
109.00
109.00
109.00
109.00
109.00
$1.0(4)$
$178.6(2)$
$-2.2(4)$
$178.9(2)$
$1.3(4)$
$-179.9(2)$
$0.9(4)$
$2.0(4)$
$-177.9(2)$
$-179.2(2)$
$178.2(2)$
$1.2(4)$
$-1.9(4)$
$-179.0(2)$

Hydrogen-bond geometry $\left(\AA,{ }^{\circ}\right)$

\begin{tabular}{lllll}
\hline$D-\mathrm{H} \cdots A$ & $D-\mathrm{H}$ & $\mathrm{H} \cdots A$ & $D \cdots A$ & $D-\mathrm{H} \cdots A$ \\
\hline $\mathrm{C} 7-\mathrm{H} 7 C \cdots \mathrm{O} 1^{\mathrm{i}}$ & 0.96 & 2.60 & $3.232(4)$ & 124 \\
\hline
\end{tabular}

Symmetry code: (i) $x-1 / 2,-y+1 / 2,-z+1$. 